\title{
The c.3040C $>\mathrm{T}$ mutation in COL1A1 is recurrent in Korean patients with infantile cortical hyperostosis (Caffey disease)
}

\author{
Tae-Joon Cho $\cdot$ Hyuk Ju Moon · Dae-Yeon Cho \\ Moon Seok Park · Dong Yeon Lee · Won Joon Yoo • \\ Chin Youb Chung $\cdot$ In Ho Choi
}

Received: 10 June 2008 / Accepted: 10 July 2008/Published online: 13 August 2008

(C) The Japan Society of Human Genetics and Springer 2008

\begin{abstract}
Infantile cortical hyperostosis (ICH) is characterized by spontaneous episodes of subperiosteal new bone formation in the long bones, mandible, and clavicle during infancy. A heterozygous missense mutation, c.3040C $>$ T (p.R1014C), in the type I collagen $\alpha 1$ chain gene (COL1A1) was reported in families with the autosomal dominant form of ICH. We examined six consecutive cases of ICH from five unrelated families and their parents. The mutation was identified in all patients and two parents tested. Our result supported that ICH is caused by the single mutation in COLIAl with incomplete penetrance.
\end{abstract}

Keywords Infantile cortical hyperostosis .

Caffey disease - Type I collagen gene

\section{Introduction}

Infantile cortical hyperostosis (ICH; Caffey disease: OMIM 114000) is characterized by fever and irritability

T.-J. Cho $(\bowtie)$ · H. J. Moon · W. J. Yoo · I. H. Choi

Department of Orthopaedic Surgery,

Seoul National University Children's Hospital,

28, Yeongeon-dong, Jongno-gu, Seoul 110-744, South Korea

e-mail: tjcho@snu.ac.kr

D.-Y. Cho

Clinical Research Institute, LabGenomics, Seoul, South Korea

M. S. Park · C. Y. Chung

Department of Orthopaedic Surgery,

Seoul National University Bundang Hospital,

Gyeonggi, South Korea

D. Y. Lee

Department of Orthopaedic Surgery,

Kangwon National University Hospital, Gangwon, South Korea along with soft tissue swelling and extensive subperiosteal reaction at the long bones, mandible, and clavicle (Caffey 1957). It usually appears before 6 months of age and spontaneously remits by 2 years of age. ICH was considered as a heritable disease because autosomal dominant inheritance has been reported in several families (Emmery et al. 1983; Gerrard et al. 1961; Newberg and Tampas 1981). A lethal type of prenatal cortical hyperostosis was described (Lecolier et al. 1992), but it seems to be a disease of a different entity from that of the autosomal dominant form. Gensure et al. (2005) performed a genome-wide mapping of the ICH gene using a family with 24 affected members and identified a heterozygous mutation of c.3040C > T; p.R1014C (according to the HUGOapproved nomenclature) in exon 41 of the type I collagen $\alpha 1$ chain gene (COL1A1). Suphapeetiporn et al. (2007) reported the same mutation in a Thai family. However, no other mutation in COL1A1 has been reported in ICH, nor has any case series of ICH with this mutation. We report the result of a mutation study for six consecutive patients with ICH from five unrelated Korean families.

\section{Materials and methods}

Six consecutive patients were diagnosed as having ICH by clinical and radiographic findings and referred to our institute from March 2002 to May 2007. There were three boys and three girls. Two of them were a brother and a sister from the same family, and the five families were not related (Table 1). Genomic DNA was isolated from peripheral leukocytes of the patients, and informed consents were obtained from the parents. Abnormal skin change as in Ehlers-Danlos type I phenotype or joint subluxation/dislocation was checked in these patients. 
Table 1 Pertinent data of six patients with infantile cortical hyperostosis

\begin{tabular}{|c|c|c|c|c|c|}
\hline Patient & Gender & Family history & Age at onset (months) & Mutation study for parents & Affected bones \\
\hline 1 & M & $(-)$ & 2 & Not tested & Right femur, both tibiae, both radii, right ulna, mandible \\
\hline 2 & M & $(+)^{\mathrm{a}}$ & 9 & Mother (-) & Both tibiae, mandible \\
\hline 3 & $\mathrm{~F}$ & $(+)^{\mathrm{a}}$ & 4 & Mother (-) & Right femur, both tibiae, both radii, both ulnae, mandible \\
\hline 4 & $\mathrm{~F}$ & $(-)$ & 1 & Not tested & Left fibula, both radii, mandible \\
\hline 5 & $\mathrm{~F}$ & $(-)$ & 2 & Father $(-)$, mother $(+)$ & Both tibiae, right radius, mandible \\
\hline 6 & M & $(-)$ & 0 & Father $(+)$, mother $(-)$ & Both tibiae \\
\hline
\end{tabular}

${ }^{a}$ Patients 2 and 3 are a brother and sister from the same family

Although we recommended the mutation study to all the parents of the five families, only five parents from three families consented to participate in this study. These parents and the patients' grandparents were interviewed, in person or by telephone, for medical history of these parents. Height was measured, joint laxity was checked using Wynne-Davis method (Wynne-Davies 1970), and abnormal skin change or joint instability was checked.

PCR was performed on genomic DNAs using primers (5'-TGA CTC CAG CGG ACT CAA GGG TCC TC- $3^{\prime}, 5^{\prime}$ GCC TCT AAG GAG GCC TGA AGA GTC CC-3') to comprise exon 41 of COLIAI as previously described (Gensure et al. 2005). The PCR product was sequenced using 3130 Genetic Analyzer (Applied Biosystems).

\section{Results}

The age at the onset of irritability and limb swelling ranged from birth to 9 months, with an average of 3 months. The tibia was affected in five cases, mandible in five, radius in three, and ulna, fibula, and clavicle in one, respectively (Table 1). All patients recovered from acute irritability, swelling, and tenderness of limbs within 4 weeks. Patient 1 , who was followed for 5 years, showed complete remodeling of the bone lesions (Fig. 1). In all patients, the heterozygous c.3040C $>\mathrm{T}$ mutation was identified.

Of the five parents tested, one mother and one father were found to carry the same mutation. They and their parents (patients' grandparents) denied any medical history suggesting ICH during infancy. Their heights were $157 \mathrm{~cm}$ $(z=-0.61)$ and $172 \mathrm{~cm}(z=-0.19)$, respectively, and they did not show any joint laxity. A cousin of the father having the mutation (patient 6) was reported to have transient irritability and lower leg swelling in infancy, but she refused to have a mutation study (Fig. 2). None of the patients and parents showed any abnormal skin change or major joint subluxation/dislocation.
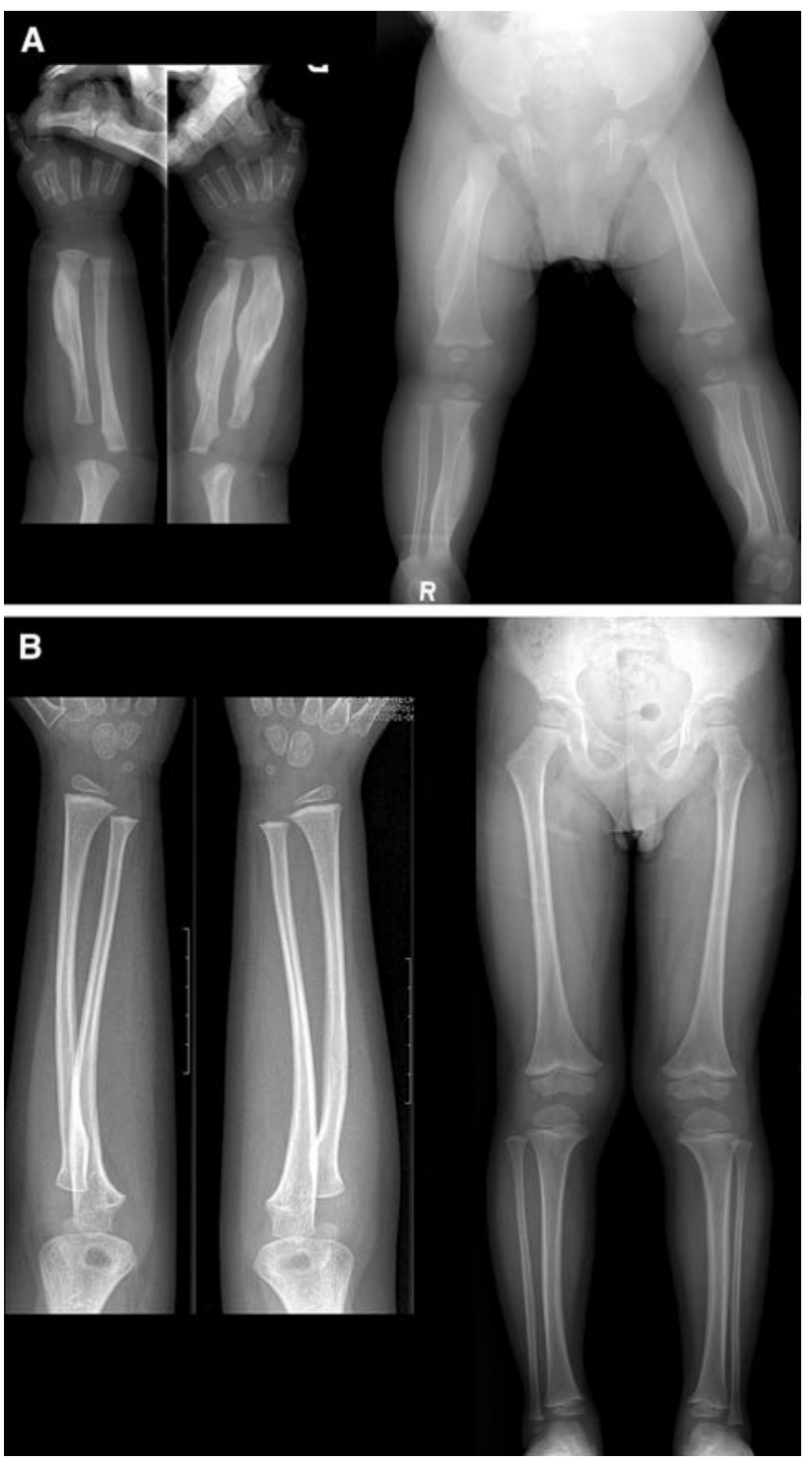

Fig. 1 Radiographs of patient 1 at age 2 months showed periosteal reactions typical of $\mathrm{ICH}$ on both tibiae, both radii, the right femur, and the right ulna (a). At age 5 years, the bony lesions were completely remodeled without any residual abnormalities (b) 


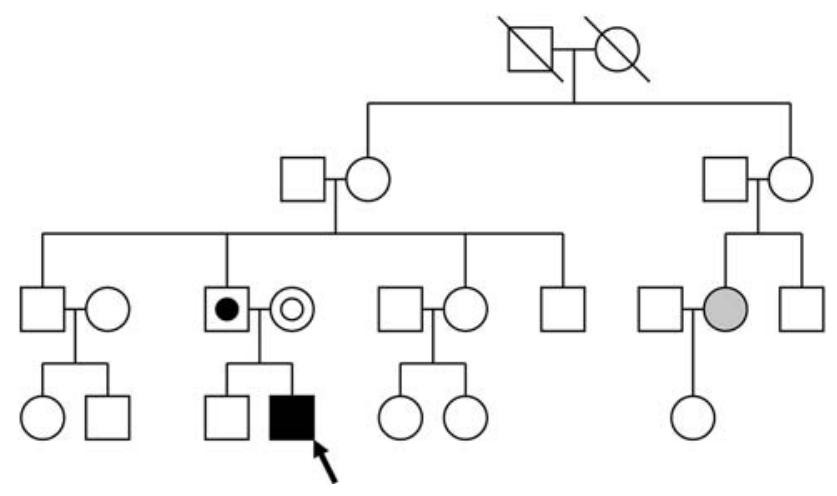

Fig. 2 Pedigree of the family of patient 6. Black symbol, affected individual; white symbols, no symptoms according to history; gray symbol, positive history suggesting ICH; black dot in the center, positive mutation study; white dot in the center, negative mutation study

Patient 4 initially had tenderness and swelling at the left lower leg at age 2 months with isolated diffuse periosteal reaction over the left fibula on a plain radiograph. Blood test showed leukocyte count of $10,160 \mathrm{~mm}^{-3}$, erythrocyte sedimentation rate of $3 \mathrm{~mm} / \mathrm{h}$ (reference $<20 \mathrm{~mm} / \mathrm{h}$ ), Creactive protein of $0.07 \mathrm{mg} / \mathrm{dl}$ (reference $<0.5 \mathrm{mg} / \mathrm{dl}$ ), and negative VDRL test. Subsequent development of periosteal reactions on radii and mandible confirmed the diagnosis of ICH.

\section{Discussion}

The authors found the same COLIAl mutation in six consecutive cases of ICH. All the parents denied any history of ICH in their infancy; however, at least two of the patients turned out to have inherited the mutation from asymptomatic parents. Gensure et al. (2005) reported that only $79 \%$ of the family members having this mutation experienced clinical manifestation of ICH. Considering the self-remitting nature of this disease, acute phase symptoms in some of the patients may not have been noticed. The phenotype of this mutation in adults also needs to be further elucidated. Some of the patients were reported to have joint laxity, but others, as in our series and previous report (Gensure et al. 2005), did not show any joint laxity.

Suphapeetiporn et al. (2007) reported on a Thai family that had an $\mathrm{ICH}$ patient without c.3040C $>\mathrm{T}$ mutation.
However, as this patient had showed only non-painful curved forearms and hallux valgus since infancy, the diagnosis is dubious. Then, all the patients certainly having ICH phenotype in two previous reports (Gensure et al. 2005; Suphapeetiporn et al. 2007) and the current study carried the same c.3040C $>\mathrm{T}$ mutation in COL1A1. It is interesting to note that only a single mutation of this large gene causes a specific ICH phenotype. Although identification of the causative mutation of this interesting disease has enormous importance for understanding its pathogenesis, further investigation is still needed to clarify the mechanism by which this genotype causes ICH.

Acknowledgments This study was supported by KOHTERF-200802 from Korean Human Technology Research Foundation.

\section{References}

Caffey J (1957) Infantile cortical hyperostosis; a review of the clinical and radiographic features. Proc R Soc Med 50:347-354

Emmery L, Timmermans J, Christens J, Fryns JP (1983) Familial infantile cortical hyperostosis. Eur J Pediatr 141:56-58

Frana L, Sekanina M (1976) Infantile cortical hyperostosis. Arch Dis Child 51:589-595

Gensure RC, Makitie O, Barclay C, Chan C, Depalma SR, Bastepe M, Abuzahra H, Couper R, Mundlos S, Sillence D, Ala Kokko L, Seidman JG, Cole WG, Juppner H (2005) A novel COL1A1 mutation in infantile cortical hyperostosis (Caffey disease) expands the spectrum of collagen-related disorders. J Clin Invest 115:1250-1257

Gerrard JW, Holman GH, Gorman AA, Morrow IH (1961) Familial infantile cortical hyperostosis. J Pediatr 59:543-548

Lecolier B, Bercau G, Gonzales M, Afriat R, Rambaud D, Mulliez N, de Kermadec S (1992) Radiographic, haematological, and biochemical findings in a fetus with Caffey disease. Prenat Diagn 12:637-641

Newberg AH, Tampas JP (1981) Familial infantile cortical hyperostosis: an update. AJR Am J Roentgenol 137:93-96

Pickering D, Cuddigan B (1969) Infantile cortical hyperostosis associated with thrombocythaemia. Lancet 2:464-465

Schweiger S, Chaoui R, Tennstedt C, Lehmann K, Mundlos S, Tinschert S (2003) Antenatal onset of cortical hyperostosis (Caffey disease): case report and review. Am J Med Genet A 120A:547-552

Suphapeetiporn K, Tongkobpetch S, Mahayosnond A, Shotelersuk V (2007) Expanding the phenotypic spectrum of Caffey disease. Clin Genet 71:280-284

Wynne-Davies R (1970) Acetabular dysplasia and familial joint laxity: two etiological factors in congenital dislocation of the hip. A review of 589 patients and their families. J Bone Joint Surg Br 52:704-716 